\title{
Early Echocardiographic Changes After Percutaneous Implantation of the Edwards SAPIEN Transcatheter Heart Valve in the Pulmonary Position
}

\author{
Shahryar M. Chowdhury, M.D. ${ }^{\star}$, Ziyad M. Hijazi, M.D. ${ }^{\dagger}$, John Rhodes, M.D. ${ }^{\ddagger}$, Saibal Kar, \\ M.D.§, Raj Makkar, M.D.§, Michael Mullen, M.D.ף, Qi-Ling Cao, M.D.†, Lydia King, Ph.D.*, \\ Jodi Akin, M.S.N.II, and Girish Shirali, M.B.B.S.\# \\ "Department of Pediatrics, Medical University of South Carolina, Charleston, South Carolina \\ ${ }^{\dagger}$ Rush Center for Congenital \& Structural Heart Disease, Rush University Medical Center, \\ Chicago, Illinois \\ ¥Department of Pediatrics, Duke University Medical Center, Durham, North Carolina \\ §Department of Medicine, Cedar Sinai Medical Center, Los Angeles, California \\ IDepartment of Medicine, The Heart Hospital, London, United Kingdom \\ "Edwards Lifesciences, Irvine, California \\ \#The Ward Family Heart Center, Children's Mercy Hospital, Kansas City, Missouri
}

\begin{abstract}
Objectives-To evaluate echocardiographic changes after SAPIEN valve implantation in the pulmonary position.
\end{abstract}

Background-The feasibility of the SAPIEN transcatheter pulmonary valve (TPV) has recently been demonstrated. We evaluated changes in pulmonary valve function and the right ventricle after SAPIEN TPV placement.

\begin{abstract}
Methods-We evaluated echocardiograms at baseline, discharge, 1 and 6 months after TPV placement in 33 patients from 4 centers. Pulmonary insufficiency severity was graded $0-4$. TPV peak and mean gradients were measured. Right ventricular (RV) size and function were quantified using routine measures derived from color, spectral, and tissue Doppler indices and twodimensional echocardiography.
\end{abstract}

\begin{abstract}
Results-At baseline, 94\% patients demonstrated pulmonary insufficiency grade $2-4$. This decreased to $12 \%$ patients at 6 months $(\mathrm{P}<0.01)$. TPV peak $(\mathrm{P}<0.01)$ and mean gradient $(\mathrm{P}<$ $0.01)$ decreased. RV end-diastolic area indexed to body surface area (BSA) $(\mathrm{P}<0.01)$, Tricuspid regurgitation (TR) gradient $(\mathrm{P}<0.01)$, and the ratio of TR jet area to BSA $(\mathrm{P}<0.01)$ decreased. Tricuspid inflow peak E:A, tissue Doppler imaging (TDI): septal E' and A', TDI: tricuspid A'
\end{abstract}

\section{(C) 2013, Wiley Periodicals, Inc.}

Address for correspondence and reprint request: Shahryar Chowdhury, M.D., 165 Ashley Ave, MSC 915, Charleston, SC 29425. Fax: (843)-792-5878; chowdhur@musc.edu.

Disclosure:

Dr. Shirali is a consultant for, and a recipient of research grants from Edwards Lifesciences. Dr. Hijazi was a consultant for Edwards Lifesciences. Dr. Kar receives research grants and honoraria from and is a consultant to Abbott Vascular. Dr. Mullen is a proctor for Edwards Lifesciences. Dr. Makkar receives consultancy fees, grant support and lectures fees from Abbott, Medtronic, and Lilly, and grant support from Johnson \& Johnson and St Jude Medical. Ms. Akin is an employee of Edwards Lifesciences. All other authors have reported that they have no relationships to disclose. 
improved between baseline and discharge, but trended back to baseline by 6-month follow-up. Tricuspid valve annulus $z$-score, $\mathrm{RV}$ area change, tricuspid annular plane systolic excursion (TAPSE), RV dP/dt, tricuspid E:E', and TDI: tricuspid annulus E' showed no change.

Conclusion-Improvements in pulmonary insufficiency and stenosis, RV size, and TR gradient and severity are seen after SAPIEN TPV placement. Selected indices of RV diastolic function improve immediately after TPV implantation, but return to baseline by 6 months. RV systolic function is unchanged.

\section{Keywords}

pulmonary valve; right ventricle; percutaneous intervention; echocardiography; congenital heart disease; pulmonary regurgitation

Patients with congenital heart disease who require right ventricle to pulmonary artery (RVPA) conduit placement often develop valve dysfunction over time. ${ }^{1}$ The pulmonary regurgitation and/or stenosis that develop may lead to right ventricle (RV) pressure and volume loading resulting in RV dilation and dysfunction, arrhythmias, tricuspid regurgitation (TR), heart failure symptoms, and ultimately, death. ${ }^{2-4}$ Surgical pulmonary valve replacement is highly effective in eliminating or greatly reducing pulmonary regurgitation ${ }^{5}$ and has low operative mortality. ${ }^{6,7}$ However, the recovery of RV function has been variable. ${ }^{8,9}$

With the advent of transcatheter pulmonary valve (TPV) implantation, a new option to replace dysfunctional pulmonary valves in RV-PA conduits is now available. The Edwards SAPIEN transcatheter heart valve (Edwards Lifesciences, Irvine, CA, USA), a trileaflet bovine pericardial valve within a balloon-expandable, stainless-steel support frame, has been shown to be safe and effective in the aortic position when implanted in elderly patients who are high risk surgical candidates. ${ }^{10}$ It has recently received Federal Drug Administration approval for inoperable patients with severe aortic valve stenosis. The COngenital Multicenter trial of Pulmonic vAlve regurgitation Studying the SAPIEN interventIONal transcatheter heart valve (COMPASSION) is a prospective, nonrandomized, multicenter study to assess the safety and efficacy of the SAPIEN transcatheter heart valve for the treatment of dysfunctional RV-PA conduits. Early phase 1 results have shown good feasibility, effectiveness, and safety. ${ }^{11}$ Our objective was to evaluate echocardiographic changes in this group after SAPIEN transcatheter heart valve implantation in the pulmonary position. We hypothesized that after percutaneous pulmonary valve placement echocardiographic measurements of RV size and TR will decrease while measures of RV function will remain unchanged. The Institutional Review Board in each participating institution approved the trial.

\section{Methods}

\section{Patient Population}

Patients were enrolled prospectively from 4 participating centers. Inclusion criteria included: (1) Weight equal to or exceeding 35 kilograms. (2) In situ conduit size of $\geq 16 \mathrm{~mm}$ and $\preceq 24$ $\mathrm{mm}$ in diameter. (3) Moderate or severe pulmonary regurgitation defined as $23+$ pulmonary regurgitation by transthoracic echocardiogram (TTE), or RV-PA conduit obstruction with a mean gradient of $>35 \mathrm{mmHg}$ by TTE. (4) Symptoms as evidenced by cardiopulmonary exercise testing. Informed consent was obtained from all potential subjects and/or their legal guardians. 


\section{Procedure}

The protocol for valve implantation has been reported previously ${ }^{11}$ and is summarized here for convenience. Procedures were performed under general anesthesia with biplane fluoroscopic guidance. After hemodynamic assessment, the minimum diameter of the conduit was assessed by angiography. Aortic root angiography or selective coronary angiography to assess for possible coronary compression was performed with simultaneous inflation of a noncompliant balloon in the conduit before RV outflow tract intervention. Pres-tenting of the conduit with a bare metal stent was performed, either in a separate procedure or during the valve implantation procedure. A $23 \mathrm{~mm}$ or $26 \mathrm{~mm}$ SAPIEN transcatheter heart valve was then implanted over a stiff guidewire and expanded via balloon inflation. After TPV placement, pressure measurements, and RV outflow tract angiography were repeated.

\section{Echocardiographic Protocol}

The echocardiography core laboratory developed the image acquisition protocol and provided on-site or web-based training to the local SAPIEN TPV implantation sites. TTEs were performed at baseline prior to TPV implantation, prior to discharge after TPV implantation, 30-day follow-up, and 6-month follow-up. All studies were performed under baseline physiologic conditions, not under the influence of anesthesia. Echocardiograms were performed by experienced sonographers at each center following a protocol which included a complete set of standardized views to evaluate the RV. These included parasternal long-axis, parasternal short-axis, apical four-chamber, RV focused apical fourchamber, and subcostal views. All echocardiograms were then sent to the core lab where they were stored digitally, databased and networked using Xcelera (Philips Medical Systems, Andover, MA, USA). All core laboratory measurements were made off line by a single reviewer and averaged over 3 beats.

\section{Evaluation of Pulmonary Valve Function}

Assessment of pulmonary valve stenosis and regurgitation were made primarily from parasternal short-axis, long-axis, and apical windows. Net peak and mean gradient through the pulmonary valve was measured by subtracting the continuous Doppler gradient through the valve from the pulse wave Doppler gradient obtained in the RV just proximal to the stent. Pressure half-time of pulmonary regurgitation was measured on patients whose regurgitation provided adequate envelopes. Pulmonary regurgitation was graded from 0 to 4 using the criteria in Figure 1. We graded pulmonary regurgitation based on jet length and jet entrance into the RV body - easily reproducible measures to compare over time. ${ }^{12,13}$ Pulmonary stenosis was graded from none to severe using the criteria in Figure 2. ${ }^{14}$

\section{Evaluation of RV Size and Tricuspid Valve Function}

RV end-diastolic area, end-systolic area, tricuspid valve annular diameter, and TR gradient were obtained from an RV centered apical four-chamber window. Tricuspid valve regurgitant orifice area was calculated from measurements in the apical four-chamber and parasternal short-axis windows.

\section{Evaluation of RV Systolic Function}

$\mathrm{RV}$ fractional area change (FAC) was defined as ([end-diastolic area - end-systolic area]/ end-diastolic area) $\times 100$. The rate of $\mathrm{RV}$ pressure rise $(\mathrm{RV} \mathrm{dP} / \mathrm{dt})$ was measured from the ascending limb of the TR continuous-wave Doppler signal between $100 \mathrm{~m} / \mathrm{sec}$ and $300 \mathrm{~m} /$ sec. From a standard apical four-chamber window, tricuspid annular plane systolic excursion (TAPSE) was obtained, and right ventricular longitudinal shortening (RV LS) was calculated as ([RV end-diastolic length - RV end-systolic length]/RV end-diastolic length). 
Pulsed tissue Doppler imaging (TDI) $\mathrm{S}^{\prime}$ velocities at the tricuspid valve annulus and interventricular septum were obtained from the apical four-chamber view.

\section{Evaluation of RV Diastolic Function}

Doppler velocities of the transtricuspid flow (E and A) were obtained from an apical fourchamber window. Tissue Doppler velocities of the tricuspid annulus and septum ( $\mathrm{E}^{\prime}$ and $\left.\mathrm{A}^{\prime}\right)$ were obtained. Derived ratios (E:A, E:E') were calculated.

\section{Statistical Analysis}

Statistics were analyzed using SAS, version 9.3 (SAS Institute, Cary, NC, USA). For continuous variables, individual paired $t$-tests were conducted on all possible pairs. To determine the trend from time 1 to time 4, repeated measures ANOVA was conducted on all individuals with measurements for each of the 4 time points. We did not impute missing data as numbers were sufficient to conduct appropriate analyses. The mean $\pm \mathrm{SD}$ was calculated for each continuous variable. For the categorical variables, a Friedman's chisquare test was used to determine whether there was a trend towards improved measures from time 1 to time 4 . A P-value $<0.05$ was considered significant.

\section{Results}

\section{Patient Population}

Thirty-three consecutive patients from 4 centers had successful SAPIEN TPV implantation in the pulmonary position between April 2008 and May 2010. Demographic data are presented in Table I. All patients underwent prestenting prior to SAPIEN valve placement. The core lab interpreted 129 echocardiograms in these 33 patients.

\section{Pulmonary Valve Function, Right Ventricular Size, and Tricuspid Valve Function}

Comprehensive results are presented in Table II. The number of patients with pulmonary regurgitation grade 2 or greater decreased from 31 of $33(94 \%)$ at baseline, to 4 of $33(12 \%)$ patients at 6 months $(\mathrm{P}<0.01)$ (Fig. 1). At the 6-month follow-up, all but 1 patient had improved pulmonary regurgitation when compared with baseline. Baseline conduit stenosis severity was moderate or greater in 20 of $33(60 \%)$ patients. This decreased to 4 of $33(12 \%)$ patients at 6 months $(\mathrm{P}<0.02)$ (Fig. 2). Of the 26 patients with mild or greater conduit stenosis, $20(77 \%)$ demonstrated improvement, while $6(23 \%)$ patients had no change in conduit stenosis severity. Two patients with no stenosis at baseline had mild stenosis at 6 months.

$\mathrm{RV}$ end-diastolic area indexed to body surface area (BSA) did not change from baseline to discharge, but decreased significantly at 30-day follow-up; it remained lower than baseline at 6 months. Similarly, there was no change in RV end systolic area between baseline and discharge with a statistically significant decrease at 30-day follow-up.

The tricuspid annulus $z$-score did not change over the follow-up period.

TR peak gradient decreased from $56 \pm 21 \mathrm{mmHg}$ at baseline to $40 \pm 12 \mathrm{mmHg}$ at 6 -month follow-up $(\mathrm{P}<0.01)$. TR severity, measured by the TR jet area indexed to BSA, decreased immediately from baseline to discharge $(\mathrm{P}<0.01)$ and remained unchanged from discharge at 6-month follow-up.

\section{Right Ventricular Function}

Comprehensive results are presented in Table III. 
RV FAC $(\mathrm{P}=0.86)$, RV LS $(\mathrm{P}=0.30)$, and RV dP/dt $(\mathrm{P}=0.52)$ showed no significant interval changes. TDI tricuspid annulus $\mathrm{S}^{\prime}$ significantly improved at discharge $(\mathrm{P}<0.05)$, but then significantly decreased at 30-day and 6-month follow-up so that at 6 months, it was not significantly different from the baseline measurement. TDI septal $S^{\prime}$ followed a similar pattern.

Tricuspid inflow peak E velocity did not significantly change through any time points. Tricuspid inflow peak A velocity significantly increased at discharge $(P<0.05)$, but then significantly decreased at 6 -month follow-up $(\mathrm{P}<0.01)$. Tricuspid inflow peak velocity E:A significantly changed from baseline to discharge $(\mathrm{P}<0.01)$, but showed no changes between baseline versus 30-day and 6-month follow-up $(\mathrm{P}=0.15)$.

TDI tricuspid annulus $\mathrm{E}^{\prime}$ and $\mathrm{E}: \mathrm{E}^{\prime}$ showed no significant change. TDI tricuspid annulus $\mathrm{A}^{\prime}$ significantly changed from baseline to discharge $(\mathrm{P}<0.01)$, but showed no changes between baseline versus 30-day and 6-month follow-up $(\mathrm{P}=0.15)$.

\section{Discussion}

The function of the pulmonary valve improved dramatically after placement of the Edwards SAPIEN TPV; this improvement was durable throughout the follow-up period. All patients had improved pulmonary regurgitation and stenosis at discharge. These improvements were comparable to or more favorable than to those seen after surgical replacement, where conduit failure rates ranged from 3 to $7 \%$ at 1 year. ${ }^{15,16}$ Comparisons with the Melody valve were also favorable. ${ }^{17}$

\section{RV Size}

We chose RV end-diastolic area as a two-dimensional echocardiographic measure of RV size because it correlates strongly with magnetic resonance imaging (MRI)-derived RV size. ${ }^{18} \mathrm{We}$ found statistically significant decreases in RV end-diastolic and end-systolic area following TPV placement. This is in line with the decreases in RV volume after TPV implantation reported by other groups. ${ }^{19-21} \mathrm{RV}$ end-diastolic and systolic area decreased between discharge and 30-day follow-up and was sustained through 6-month follow-up. However, there was no further evidence of decreases in ventricular size between 30-day and 6-month follow-up suggesting changes in RV size occur early after TPV implantation.

\section{RV Systolic Function}

We used RV FAC and TAPSE-derived RV longitudinal shortening to quantify RV systolic function. RV FAC has been shown to correlate with RV ejection fraction (EF) by MRI, 22,23 and is an independent predictor of mortality after pulmonary embolism ${ }^{24}$ and myocardial infarction. ${ }^{25}$ TAPSE has correlated strongly with radionuclide angiography, biplane Simpson RV EF, and RV FAC. ${ }^{26-28}$ These measures were depressed at baseline in our patients. We found no changes in global measures of RV systolic function after TPV placement.

Almost all of our study population had mixed pulmonary regurgitation and stenosis, or pulmonary regurgitation alone. After TPV, this population has been shown to have no change in RV EF by MRI, similar to our findings. ${ }^{20,29,30}$ Patients undergoing surgical pulmonary valve replacement for severe pulmonary regurgitation exhibit similar patterns. ${ }^{8,15,31-33}$ Coats et al. ${ }^{29}$ analyzed RV function by MRI in patients with only pulmonic stenosis undergoing TPV placement and found improvement in systolic function. We only enrolled 2 patients with isolated stenosis, making a subgroup analysis impossible. 
Regional measures of RV function improved at discharge, but returned to baseline at 30-day follow-up showing no sustained change. This is in contrast with other studies that have shown no change in TDI measures of systolic function after TPV in patients with pulmonary regurgitation. ${ }^{29,34}$ Improvements in 2D RV strain by speckle tracking have been seen in patients receiving Melody valves whose preimplantation pulmonary valve dysfunction was similar to that encountered in our cohort. ${ }^{35}$ However, these patients were only evaluated before and immediately after implantation, so it is not known if improvements in strain were sustained over short-term follow-up. Perhaps the mechanism which allows patients with pure pulmonic stenosis to recover systolic function is retained in the population with mixed disease, allowing them some mild nonsustained recovery of RV systolic function.

Interestingly, regional measures of systolic function have been shown to actually worsen after surgical pulmonary valve replacement. ${ }^{36}$ However, this may be associated with cardiopulmonary bypass or other confounders, and the results may not be comparable to our population.

\section{RV Diastolic Function}

Changes in RV diastolic function following pulmonary valve replacement are sparsely reported. Our patients showed an increased TDI tricuspid A', septal E' and A', and an increase in Doppler A velocity at discharge. It is conceivable that the increased Doppler A velocity is actually a marker of improved diastolic function from a "pseudo-normal" state. These changes all returned to baseline at 30-day follow-up. Coats et al. ${ }^{29}$ found no changes in TDI tricuspid velocities after TPV. However, Frigiola et al. ${ }^{16}$ found an increase in TDI tricuspid $\mathrm{E}^{\prime}$ after surgical pulmonary valve replacement in patients with RV volumes $<150$ $\mathrm{mL} / \mathrm{m}^{2}$. This suggests recovery of $\mathrm{RV}$ diastolic function may be possible if valve replacement occurs before RV dilation reaches a threshold precluding recovery.

\section{RV Remodeling}

The improvements acutely seen after TPV, including pulmonary regurgitation grade, conduit gradients, RV size, and TR severity persisted through the 30-day and 6-month follow-up. However, no measure significantly improved between 30 days and 6 months. Longer term follow-up may be needed to detect further signs of ventricular remodeling; however, Lurz et al. ${ }^{37}$ reported a similar lack of improvement even after 2 years of follow-up. This, along with the lack of improvement in ventricular function, may suggest that patients with chronic $\mathrm{RV}$ volume overload suffer from irreversible RV remodeling and/or damage to myocardium. While this brings into question the utility of pulmonary valve replacement, insertion of the TPV may well have arrested worsening of RV size and function in our patients. Our findings suggest that long-standing pulmonary regurgitation is a more important contributor to patient morbidity than once thought ${ }^{38}$ and earlier pulmonary valve replacement should be considered after the onset of significant regurgitation. Ideally, a randomized, controlled trial should be performed to determine the optimal timing/utility of TPV placement in this population.

\section{Postimplantation Imaging Protocol}

Most studies investigating RV changes after surgical pulmonary valve replacement use MRI as the modality of choice when assessing RV size and function. ${ }^{7}$ With the increasing implantation rate of TPVs, the use of MRI is becoming difficult. As previously reported by Kenny et al., ${ }^{11}>50 \%$ of our cohort had uninterpretable MRIs at follow-up for RV volumes, $\mathrm{EF}$, and regurgitant fraction due to artifact from the stainless steel stent. Studies using the Melody valve have shown comparable problems with MRI data. ${ }^{19}$ Our results using transthoracic echocardiography show similar trends when assessing pulmonary valve function, RV size, and RV function as have been shown with MRI after TPV implantation. Echocardiography becomes very useful in this setting and may be the imaging modality of 
choice. This may become increasingly true as the age of TPV recipients decreases over the next few years, offering a safe alternative to MRI and the risks that come with the associated anesthesia. Utilization of imaging protocols will be important to detect changes in echo findings over time.

\section{Limitations}

While the measurements we included in our protocol were carefully chosen, echocardiographic analysis of changes in the right heart has inherent limitations. There are no validated standards for quantification of pulmonary regurgitation. The pulmonary regurgitation jet length, for example, is affected by the driving pressure gradient between the pulmonary artery and the RV, making it less reliable when comparing patients with differing pulmonary artery pressures. ${ }^{39}$ Measurement of the pulmonary stenosis peak instantaneous gradient is Doppler angle dependent, inaccurate if more than one area of stenosis is present in a series and may be overestimated when compared with the invasive catheterization peak to peak gradient. ${ }^{14}$ Quantification of TR by jet area correlates well with angiography, however, quantification of eccentric jets may underestimate severity. ${ }^{39}$ Tissue Doppler measures of systolic and diastolic function are angle dependent and assume that a regional measure accurately represents the function of the entire ventricle. ${ }^{40}$ As TPV technology advances to the point where these valves can be placed in transannular patches, it will be important to note that measurements of RV area from the apical four-chamber view are inherently limited because they do not include the area contained in the outflow tract. This may limit our ability to evaluate RV size and function in patients who had a transannular patch because a significant portion of their RV volume may be contained in their outflow tract and the effects of the patch on RV function will not be able to be assessed, resulting in underestimation of RV size/volume..$^{40}$ Measuring RV size from other echocardiographic views may add additional useful information in future protocols. ${ }^{41}$

Although we have reported immediate benefits of the TPV with early-term follow-up, longer term studies will be needed to determine longevity of the SAPIEN TPV compared with surgical interventions. We will provide longer term results as we include more participating institutions and enroll more patients. This may help identify long-term and/or small changes in ventricular remodeling. Because our patients were heterogeneous in terms of the nature of right ventricular outflow tract flow perturbation, we were not able to examine the potentially differing responses of volume versus pressure load relief on RV function in these patients.

\section{Conclusions}

Follow-up after SAPIEN TPV placement revealed improvements in pulmonary regurgitation and stenosis, RV size, and TR gradient and severity. These changes were seen immediately and were sustained over 6 months. Select indices of RV diastolic function improved immediately after TPV implantation, but returned to baseline by 6 months. Global measures of RV systolic function remained unchanged. COMPASSION will follow these patients for 5 years to assess the long-term functional outcome of the SAPIEN TPV when placed in the pulmonary position. Long-term changes in the RV after percutaneous pulmonary valve implantation will also be assessed.

\section{Acknowledgments}

Sources of Funding: This trial was sponsored and funded through Edwards Lifesciences LLC, Irvine, CA. 


\section{References}

1. Caldarone CA, McCrindle BW, Van Arsdell GS, et al. Independent factors associated with longevity of prosthetic pulmonary valves and valved conduits. J Thorac Cardiovasc Surg. 2000; 120:1022-1031. [PubMed: 11088021]

2. Murphy JG, Gersh BJ, Mair DD, et al. Long-term outcome in patients undergoing surgical repair of tetralogy of Fallot. N Engl J Med. 1993; 329:593-599. [PubMed: 7688102]

3. Nollert G, Fischlein T, Bouterwek S, et al. Longterm survival in patients with repair of tetralogy of Fallot: 36-year follow-up of 490 survivors of the first year after surgical repair. J Am Coll Cardiol. 1997; 30:1374-1383. [PubMed: 9350942]

4. Geva T, Sandweiss BM, Gauvreau K, et al. Factors associated with impaired clinical status in longterm survivors of tetralogy of Fallot repair evaluated by magnetic resonance imaging. J Am Coll Cardiol. 2004; 43:1068-1074. [PubMed: 15028368]

5. Cheung EW, Wong WH, Cheung YF. Meta-analysis of pulmonary valve replacement after operative repair of tetralogy of Fallot. Am J Cardiol. 2010; 106:552-557. [PubMed: 20691315]

6. Kanter KR, Budde JM, Parks WJ, et al. One hundred pulmonary valve replacements in children after relief of right ventricular outflow tract obstruction. Ann Thorac Surg. 2002; 73:1801-1806. discussion 1806-7. [PubMed: 12078772]

7. Oosterhof T, Meijboom FJ, Vliegen HW, et al. Long-term follow-up of homograft function after pulmonary valve replacement in patients with tetralogy of Fallot. Eur Heart J. 2006; 27:1478-1484. [PubMed: 16707545]

8. Vliegen HW, van Straten A, de Roos A, et al. Magnetic resonance imaging to assess the haemodynamic effects of pulmonary valve replacement in adults late after repair of tetralogy of Fallot. Circulation. 2002; 106:1703-1707. [PubMed: 12270866]

9. Therrien J, Siu SC, McLaughlin PR, et al. Pulmonary valve replacement in adults late after repair of tetralogy of Fallot: Are we operating too late? J Am Coll Cardiol. 2000; 36:1670-1675. [PubMed: 11079675]

10. Leon MB, Smith CR, Mack M, et al. Transcatheter aortic-valve implantation for aortic stenosis in patients who cannot undergo surgery. N Engl J Med. 2010; 363:1597-1607. [PubMed: 20961243]

11. Kenny D, Hijazi ZM, Kar S, et al. Percutaneous implantation of the Edwards SAPIEN transcatheter heart valve for conduit failure in the pulmonary position: Early phase 1 results from an international multicenter clinical trial. J Am Coll Cardiol. 2011; 58:2248-2256. [PubMed: 22078433]

12. Takao S, Miyatake K, Izumi S, et al. Clinical implications of pulmonary regurgitation in healthy individuals: Detection by cross sectional pulsed Doppler echocardiography. Br Heart J. 1988; 59:542-550. [PubMed: 3382565]

13. Feigenbaum, H.; Armstrong, WF.; Ryan, T. Echocardiography. 6. Philadelphia: Lippincott Williams \& Wilkens; 2005.

14. Allen HD, Beekman RH III, Garson A Jr, et al. Pediatric therapeutic cardiac catheterization: A statement for healthcare professionals from the Council on Cardiovascular Disease in the Young. American Heart Association. Circulation. 1998; 97:609-625. [PubMed: 9494035]

15. Oosterhof T, van Straten A, Vliegen HW, et al. Preoperative thresholds for pulmonary valve replacement in patients with corrected tetralogy of Fallot using cardiovascular magnetic resonance. Circulation. 2007; 116:545-551. [PubMed: 17620511]

16. Frigiola A, Tsang V, Bull C, et al. Biventricular response after pulmonary valve replacement for right ventricular outflow tract dysfunction: Is age a predictor of outcome? Circulation. 2008; 118:S182-S190. [PubMed: 18824753]

17. Zahn EM, Hellenbrand WE, Lock JE, et al. Implantation of the melody transcatheter pulmonary valve in patients with a dysfunctional right ventricular outflow tract conduit early results from the US clinical trial. J Am Coll Cardiol. 2009; 54:1722-1729. [PubMed: 19850214]

18. Schenk P, Globits S, Koller J, et al. Accuracy of echocardiographic right ventricular parameters in patients with different end-stage lung diseases prior to lung transplantation. J Heart Lung Transplant. 2000; 19:145-154. [PubMed: 10703690] 
19. McElhinney DB, Hellenbrand WE, Zahn EM, et al. Short-and medium-term outcomes after transcatheter pulmonary valve placement in the expanded multicenter US melody valve trial. Circulation. 2010; 122:507-516. [PubMed: 20644013]

20. Vezmar M, Chaturvedi R, Lee KJ, et al. Percutaneous pulmonary valve implantation in the young: 2-year follow-up. JACC Cardiovasc Interv. 2010; 3:439-448. [PubMed: 20398873]

21. Lurz P, Giardini A, Taylor AM, et al. Effect of altering pathologic right ventricular loading conditions by percutaneous pulmonary valve implantation on exercise capacity. Am J Cardiol. 2010; 105:721-726. [PubMed: 20185023]

22. Lai WW, Gauvreau K, Rivera ES, et al. Accuracy of guideline recommendations for twodimensional quantification of the right ventricle by echocardiography. Int J Cardiovasc Imaging. 2008; 24:691-698. [PubMed: 18438737]

23. Anavekar NS, Gerson D, Skali H, et al. Two-dimensional assessment of right ventricular function: An echocardiographic-MRI correlative study. Echocardiography. 2007; 24:452-456. [PubMed: 17456062]

24. Nass N, McConnell MV, Goldhaber SZ, et al. Recovery of regional right ventricular function after thrombolysis for pulmonary embolism. Am J Cardiol. 1999; 83:804-806. [PubMed: 10080447]

25. Anavekar NS, Skali H, Bourgoun M, et al. Usefulness of right ventricular fractional area change to predict death, heart failure, and stroke following myocardial infarction (from the VALIANT ECHO study). Am J Cardiol. 2008; 101:607-612. [PubMed: 18308007]

26. Kaul S, Tei C, Hopkins JM, et al. Assessment of right ventricular function using two-dimensional echocardiography. Am Heart J. 1984; 107:526-531. [PubMed: 6695697]

27. Lopez-Candales A, Dohi K, Rajagopalan N, et al. Defining normal variables of right ventricular size and function in pulmonary hypertension: An echocardiographic study. Postgrad Med J. 2008; 84:40-45. [PubMed: 18230750]

28. Miller D, Farah MG, Liner A, et al. The relation between quantitative right ventricular ejection fraction and indices of tricuspid annular motion and myocardial performance. J Am Soc Echocardiogr. 2004; 17:443-447. [PubMed: 15122184]

29. Coats L, Khambadkone S, Derrick G, et al. Physiological consequences of percutaneous pulmonary valve implantation: The different behaviour of volume- and pressure-overloaded ventricles. Eur Heart J. 2007; 28:1886-1893. [PubMed: 17595193]

30. Coats L, Khambadkone S, Derrick G, et al. Physiological and clinical consequences of relief of right ventricular outflow tract obstruction late after repair of congenital heart defects. Circulation. 2006; 113:2037-2044. [PubMed: 16636174]

31. Ghez O, Tsang VT, Frigiola A, et al. Right ventricular out-flow tract reconstruction for pulmonary regurgitation after repair of tetralogy of Fallot: Preliminary results. Eur J Cardiothorac Surg. 2007; 31:654-658. [PubMed: 17267236]

32. Dave HH, Buechel ER, Dodge-Khatami A, et al. Early insertion of a pulmonary valve for chronic regurgitation helps restoration of ventricular dimensions. Ann Thorac Surg. 2005; 80:1615-1620. [PubMed: 16242426]

33. Gengsakul A, Harris L, Bradley TJ, et al. The impact of pulmonary valve replacement after tetralogy of Fallot repair: A matched comparison. Eur J Cardiothorac Surg. 2007; 32:462-468. [PubMed: 17627832]

34. Kjaergaard J, Iversen KK, Vejlstrup NG, et al. Effects of chronic severe pulmonary regurgitation and percutaneous valve repair on right ventricular geometry and contractility assessed by tissue Doppler echocardiography. Echocardiography. 2010; 27:854-863. [PubMed: 20546000]

35. Moiduddin N, Asoh K, Slorach C, et al. Effect of trans-catheter pulmonary valve implantation on short-term right ventricular function as determined by two-dimensional speckle tracking strain and strain rate imaging. Am J Cardiol. 2009; 104:862-867. [PubMed: 19733725]

36. Knirsch W, Dodge-Khatami A, Kadner A, et al. Assessment of myocardial function in pediatric patients with operated tetralogy of Fallot: Preliminary results with 2D strain echocardiography. Pediatr Cardiol. 2008; 29:718-725. [PubMed: 18506501]

37. Lurz P, Nordmeyer J, Giardini A, et al. Early versus late functional outcome after successful percutaneous pulmonary valve implantation: Are the acute effects of altered right ventricular loading all we can expect? J Am Coll Cardiol. 2011; 57:724-731. [PubMed: 21292132] 
38. Katz NM, Blackstone EH, Kirklin JW, et al. Late survival and symptoms after repair of tetralogy of Fallot. Circulation. 1982; 65:403-410. [PubMed: 7053900]

39. Zoghbi WA, Enriquez-Sarano M, Foster E, et al. Recommendations for evaluation of the severity of native valvular regurgitation with two-dimensional and Doppler echocardiography. J Am Soc Echocardiogr. 2003; 16:777-802. [PubMed: 12835667]

40. Rudski LG, Lai WW, Afilalo J, et al. Guidelines for the echocardiographic assessment of the right heart in adults: A report from the American Society of Echocardiography endorsed by the European Association of Echocardiography, a registered branch of the European Society of Cardiology, and the Canadian Society of Echocardiography. J Am Soc Echocardiogr. 2010; 23:685-713. [PubMed: 20620859]

41. Chaowalit N, Durongpisitkul K, Krittayaphong R, et al. Echocardiography as a simple initial tool to assess right ventricular dimensions in patients with repaired tetralogy of Fallot before undergoing pulmonary valve replacement: Comparison with cardiovascular magnetic resonance imaging. Echocardiography. 2012; 29:1239-1246. 


\section{Change in Pulmonary Regurgitation}

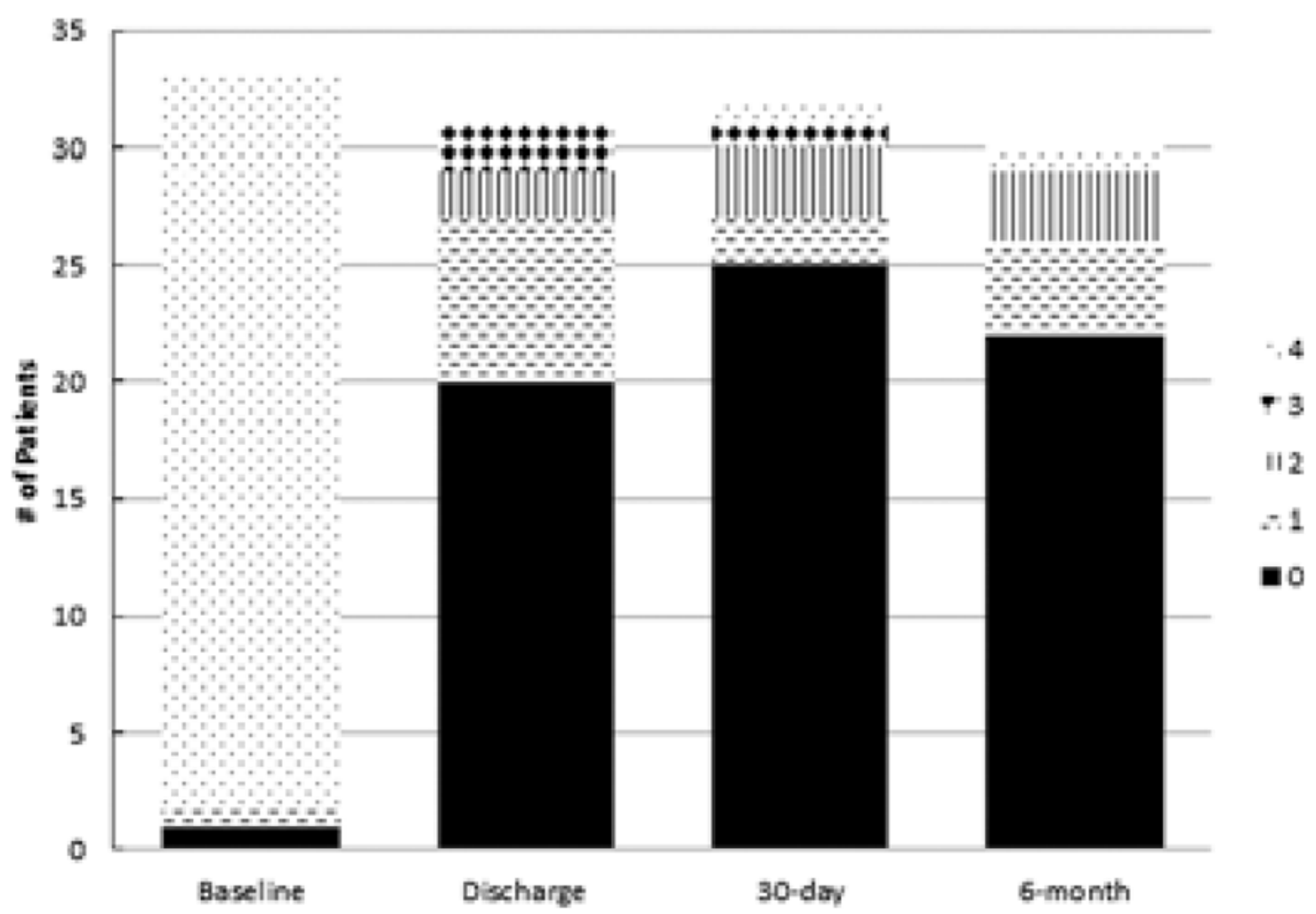

Figure 1.

Change in pulmonary regurgitation. Changes in pulmonary regurgitation over the follow-up period. Pulmonary regurgitation was graded from 0 to 4 based on regurgitant jet length and extension into the right ventricular $(\mathrm{RV})$ : $0=$ none; $1=\operatorname{trivial}(<1 \mathrm{~cm}) ; 2=$ mild $(1-2 \mathrm{~cm}) ; 3$ $=$ moderate $(>2 \mathrm{~cm}$, does not extend into RV body); or $4=$ severe $(>2 \mathrm{~cm}$, extends into $\mathrm{RV}$ body). 


\section{Change in Pulmonary Stenosis}

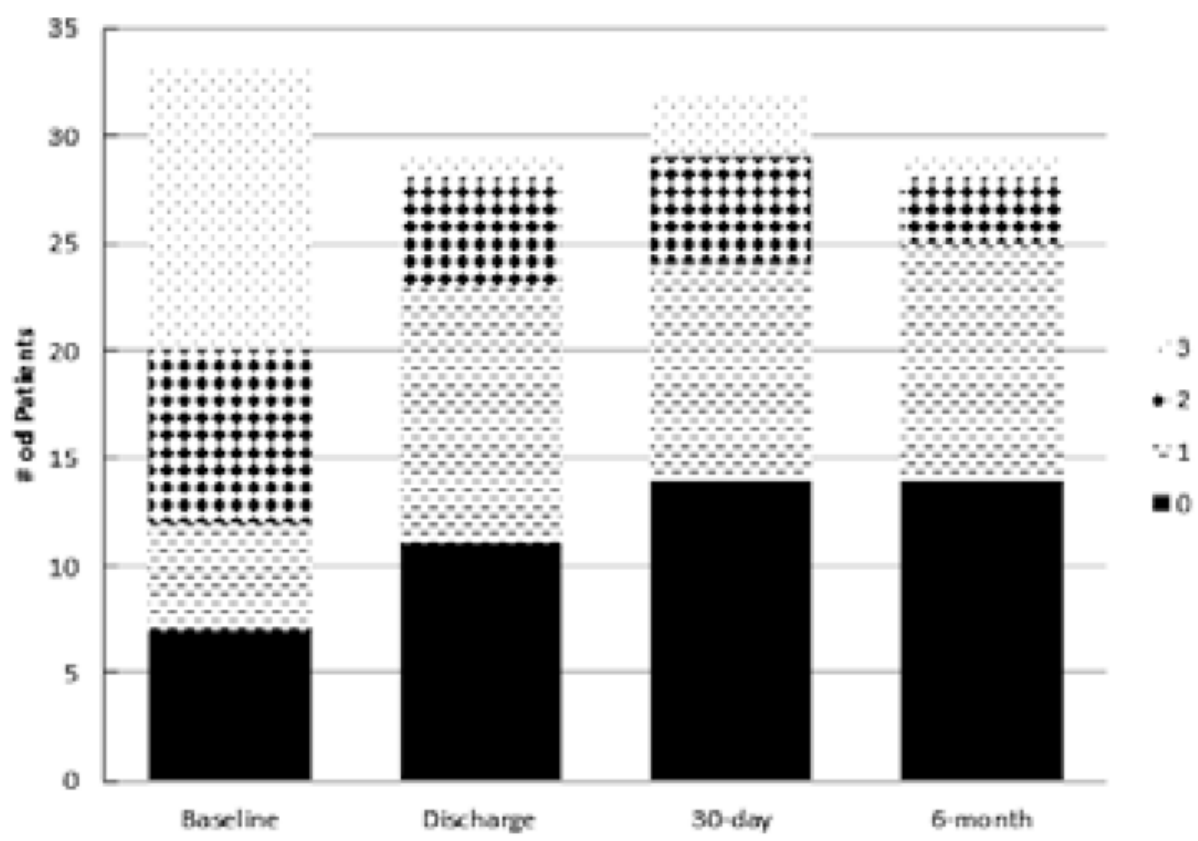

Figure 2.

Change in pulmonary stenosis. Changes in pulmonary stenosis over the follow-up period. Pulmonary stenosis was graded from 0 to 3 based on net peak gradient: $0=$ none $(<16$ $\mathrm{mmHg}) ; 1=$ mild $(16-30 \mathrm{mmHg}) ; 2=$ moderate $(31-45 \mathrm{mmHg}) ;$ or $3=$ severe $(>45$ $\mathrm{mmHg})$. 


\section{TABLE I}

Patient Demographics

\begin{tabular}{lc}
\hline Age, years & $30.3 \pm 15.1$ \\
Weight, kg & $73.4 \pm 22.9$ \\
Male/female & $24 / 12$ \\
Diagnosis & 16 \\
$\quad$ Tetralogy of Fallot & 11 \\
Ross procedure & 1 \\
Transposition of the great arteries & 8 \\
Other & $1.94(1-5)$ \\
Open heart surgeries & \\
RVOT conduit types & 29 \\
Homograft & 7 \\
Other & $23.4 \pm 3.9$ \\
Original RVOT conduit size, mm & 12 \\
Conduit dysfunction & 2 \\
Regurgitation & 19 \\
Stenosis & \\
Mixed & \\
RVOT prestenting & \\
Stent placed at time of procedure & \\
Stent placed before day of procedure & 12 \\
\hline
\end{tabular}

Values are mean $\pm \mathrm{SD}, \mathrm{n}$, or $\mathrm{n}$ (range).

RVOT $=$ right ventricular outflow tract. 


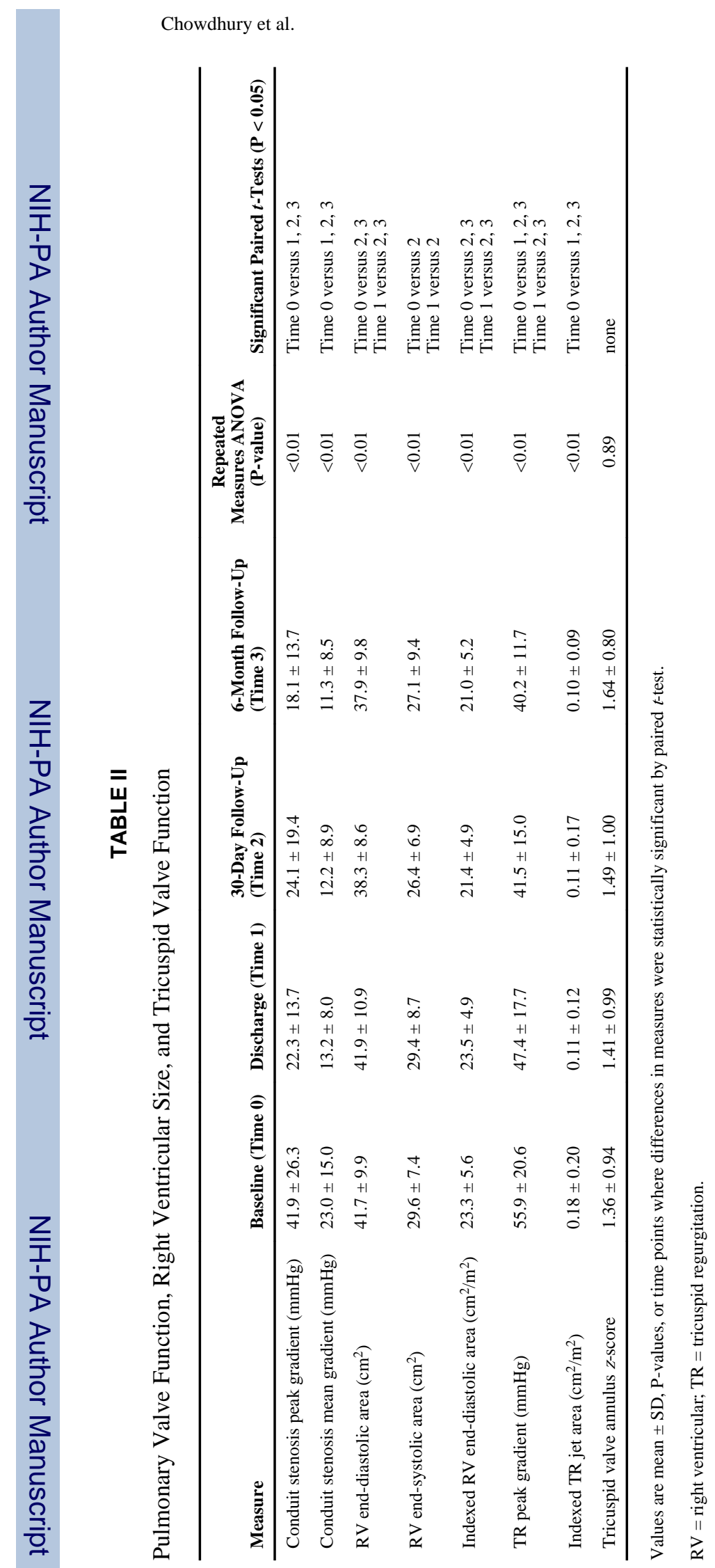

Echocardiography. Author manuscript; available in PMC 2013 November 05. 


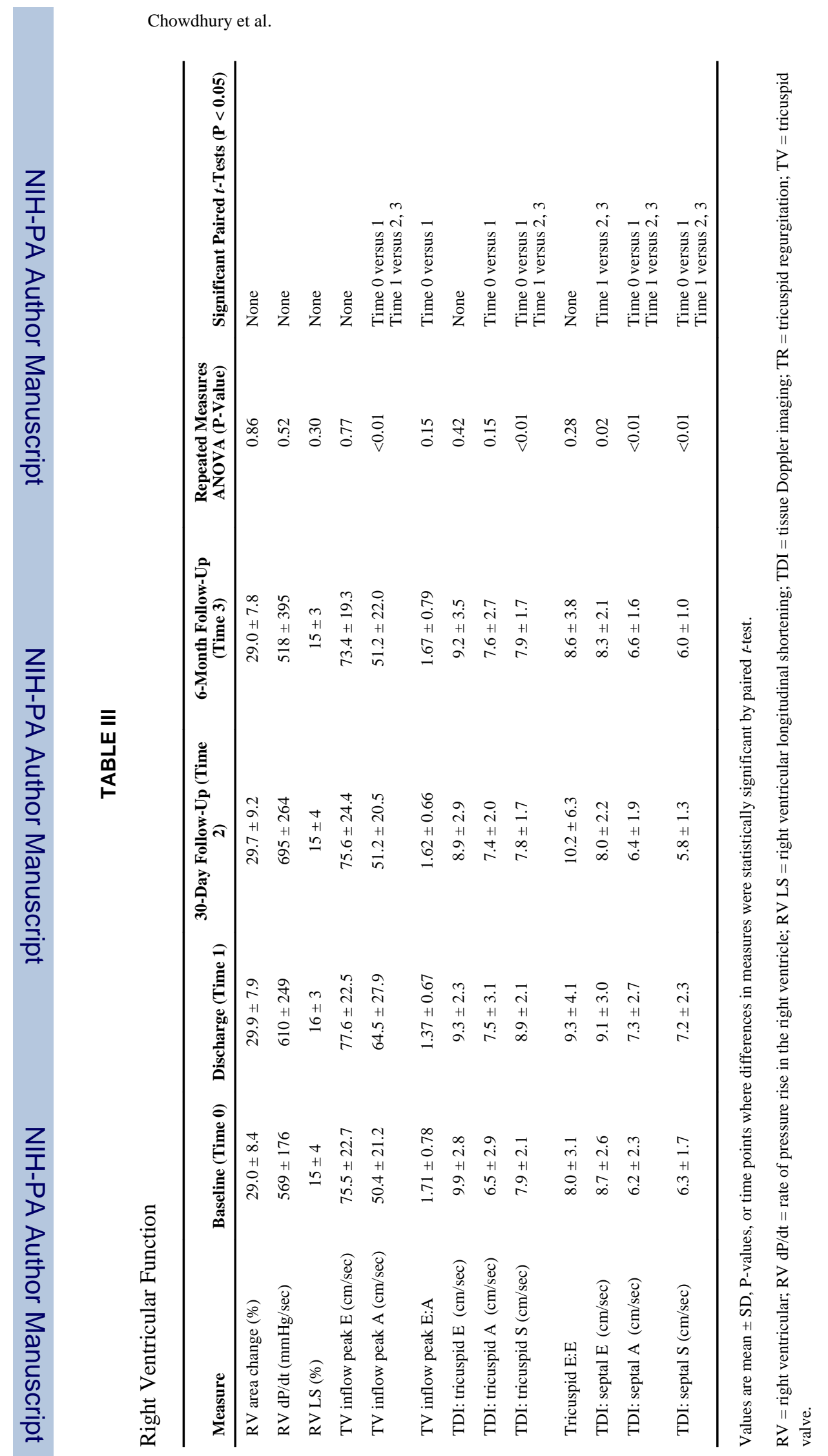

Echocardiography. Author manuscript; available in PMC 2013 November 05. 